\title{
New Developments in Pathogen Detection and Identification
}

\author{
Gustavo Nolasco \\ BioFIG / Universidade do Algarve \\ Campus de Gambelas \\ 8005-139 Faro \\ Portugal
}

Keywords : FRET, LAMP, metagenomics, microarrays, macroarrays, typing, deep sequencing, si-RNA

\begin{abstract}
By the turn of the century most of the methods for amplifying the pathogen detection signal had already been developed. However only a few were successfully incorporated into routine diagnosis schemes. At present, real time PCR became the predominant choice with signal transduction based on fluorescence generated by intercalating dyes or fluorescent resonant energy transfer. Real-time PCR is no more exclusively restricted to niches for which no alternative was feasible; its use is widening and is substituting or complementing other techniques like ELISA. On the low technology side and judging from the success in medical fields, Loop Mediated Isothermal Amplification (LAMP) appears as an interesting alternative. Convergence into a single platform led to the concept of crop-oriented diagnosis, e.g, a unique device or system that could be used in the detection of the relevant pathogens of a crop. However PCR by itself is not enough robust to support the degree of multiplexing needed and fluorescence detection systems are limited to a reduced number of dyes that can be used simultaneously. Initial attempts to develop alternative systems relied in the use of microarrays but suffer from limited sensitivity. Low Density Arrays, in which individual PCR reactions are spatially separated and done in parallel appear an interesting option, already available for grapevine. All these assays only provide an answer regarding the presence of certain pathogens for which there is an a priori suspicion. Recently introduced high massively parallel sequencing coupled to metagenomic analysis appears to be a major breakthrough in diagnosis, enabling the non-targeted diagnosis of bacteria, virus, fungi and novel agents in a single assay. These have been implemented among others for citrus and grapevine.
\end{abstract}

\section{Diversity of amplification methods}

The sequence-specific amplification of nucleic acids represented a major breakthrough in pathogen detection at the turn of the century, enabling to obtain an unprecedented level of sensitivity and a sound control over specificity.

Besides the well known PCR, a number of techniques to amplify the pathogen detection signal had already been developed by that time (Table 1). Taken together these techniques differ widely in the enzymes and kind of primers which are employed. In some of these (e.g. the Ligase Chain Reaction, LCR) the newly synthesized nucleic acid strand is, like in PCR, separated from the template by heat denaturation and thus require a thermocycler. Others, like the Nucleic Acid Sequence-Based Amplification (NASBA) or 
the Loop-mediated Isothermal Amplification (Notomi et al. 2000) - described in more detail below- use an enzymatic activity to separate the nucleic acid strands. Some of these mimic the DNA replication in vivo. The Rolling Circle Amplification is an isothermal technique that uses the DNA polymerase from Phi 29 phage and mimics the natural process of replication of the circular phage genome. The enzyme has a strand displacement activity and produces a single-stranded linear multimer of the circular molecule which is by its turn primed and serves as a template for a mass of doublestranded concatemers (Fig. 1). Other techniques rely on the use of a DNA helicase, an enzyme which unwinds the ds-DNA, and on single stranded binding proteins. The Helicase Dependent Amplification (Fig. 1) enables a continuous annealing, polymerization and strand separation like in PCR but without the need of temperature cycling (Vincent et al. 2004). A review of Helicase dependent systems can be found in Jeong et al. (2009).

Contrarily to the expectations that this diversity of techniques might anticipate, most of them never jumped from Medical or Veterinary fields to the Plant Protection fields.

\section{Implementation of PCR based methods}

At present, PCR, coupled to fluorescent real-time detection of the amplified products became the preferred choice for routine diagnosis based in nucleic acids. Probably the expiry of PCR patent in 2006 and the intrinsic robustness of the real-time PCR platform contributed to this diffusion. In what concerns pathogens of fruit tree crops, real-time PCR is no more exclusively used in restricted niches for which no alternative was feasible (e.g. phytoplasmas). Its use is widening and gradually substituting other techniques, even ELISA for some viruses.

Signal detection in real-time PCR is currently based on the fluorescence generated by intercalating dyes or, with a gain in specificity, with probes based on Fluorescent Energy Transfer. These probes are doubly labelled with fluorescent dyes which have a partial overlap of the emission and excitation spectra and can be found in a variety of formats: Taqman, Molecular Beacons, Scorpions, Lux primers etc. (Table 2). Typically the part of the probe complementary to the target has a length ranging between $20 \mathrm{nt}$ and $30 \mathrm{nt}$. For highly variable pathogens as RNA viruses it may be difficult to find conserved regions with this length. In such cases it is possible to use probes with a modified backbone e.g. Minor Groove Binding probes (Kutyavin et al. 2000), Locked Nucleic acid probes (Kennedy et al. 2006) or Peptide Nucleic acids (Ortiz et al. 1998). These modifications result in an increase of the melting temperature of the hybrid enabling to reduce the probe length to $15 \mathrm{nt}$ or less. A panoply of organic fluorescent dyes are currently offered but in the near future these may be substituted by other materials as quantum dots. Quantum dots are fluorescent inorganic semiconductor nanocrystals that have improved spectral properties that encourage their use in a number of biological labelling applications. These properties include broad excitation and size-tunable emission spectra, photo-stability and relatively high quantum yields (Algar et al. 2010). The current limitation in the use of fluorescent probes resides in the real time thermocyclers available which, at best (theroretically), are able to simultaneously distinguish among 4 to 6 different dyes.

\section{LAMP as an alternative to PCR}


Loop-mediated Isothermal Amplification, LAMP (Notomi et al. 2000) is a very interesting alternative to PCR. Among other characteristics, it has an amplification power one or two orders of magnitude higher than PCR, it can be performed in a nonsophisticated environment as it relies in an isothermal amplification (at $65^{\circ} \mathrm{C}$, it can be performed in a warm water bath) and it is more robust than PCR regarding the presence of biological contaminants (Kaneko et al. 2007). Although developed ten years ago, it started being intensively used in the medical fields three years ago (more than 200 references since than). In comparison the use of LAMP in phytodiagnostics is still incipient; nevertheless it has been applied for the detection of viruses, viroids, bacteria, fungi and nematodes (Fukuta et al. 2003; Fukuta et al. 2004; Nie 2005; Varga and James 2006; Tomlinson et al. 2007; Kubota et al. 2008; Boubourakas et al. 2009; Kikuchi et al. 2009; Kuan et al. 2010; Rigano et al. 2010; Zhao et al. 2010). The original LAMP method relies in the activity of Bst DNA polymerase, a polymerase that has a strand displacement activity, and four primers which anneal to 6 regions of the template (two of the primers are specific for more than one region). The amplification procedure is complex (see Fig. 2 and detailed schemes in Notomi et al. (2000) and Nagamine et al. (2002)) and is centred around a dumbbell-like structure which will form multimeric structures during the amplification. Typically this procedure extends for about one hour. For RNA targets a reverse transcription step can be included by adding Reverse Transcriptase to the reaction mixture and providing an initial incubation step at the appropriate temperature. Two additional primers, can also be used to increase the amplification power (Nagamine et al. 2002). The design of the primers requires some care as there should be a relationship in their relative stability and it is important to avoid self priming. Online tools help primer design (http://primerexplorer.jp/elamp4.0.0/).

To see how this technique could accommodate the natural variability of an RNA virus we designed a set of primers with the intent of detecting the five phylogenetic groups described (Gouveia et al. 2009) for Grapevine leafroll associated virus-3 (GLRaV-3). The primers target a part of the coat protein gene in which the overall diversity (single p-distance) is $6.5 \%$. Care was taken in the design to avoid mismatches close to the 3' ends of primers. It was possible to amplify samples from each phylogenetic group. Another characteristic of LAMP is the insensitiveness to the presence of plant compounds that usually inhibit molecular biology reactions. This allows its use in immunocapture assays (Fukuta et al. 2004) or even with crude plant extracts.

The high amount of DNA produced in LAMP opens new cheap and practical alternatives to monitor the results of the amplification. Visual or photographic assessment of fluorescence originated by FRET probes have been used for specific detection of LAMP products (Curtis et al. 2009). For unspecific detection of LAMP products, diverse authors have used intercalating dyes like SYBR green or monitored the changes in turbidity of the reaction originated by the precipitation of magnesium pyrophosphate (Mori et al. 2001) due to large amount of pyrophosphate originated in the polymerization reaction. These changes can be followed by a turbidimeter (Mori et al. 2004) or an ELISA plate reader at $600 \mathrm{~nm}$ (Nie 2005). Depletion of magnesium ion due to precipitation as magnesium pyrophosphate can also be colorimetrically quantified with hidroxynaphtol blue, a reagent commonly used to titrate the amount of alkaline earth ions in water (Goto et al. 2009). A lateral flow device has also been used for the detection of amplified products (Rigano et al. 2010). However, some occasional reports of unspecific amplifications (see Curtis et al. (2009)) may restrain the enthusiasm in the development of unspecific methods for monitoring the amplification. 



\section{Pathogen Typing}

Although pathogen detection is done at the species level it may in certain cases be important to identify pathogen variants (strains, serotypes, etc..) that differ in the potential to cause disease. If data regarding the genomic variability of the pathogen is available and can be related to epidemiological characteristics an array of probes can be used for genotyping instead of individual typing reactions. Microarrays have been developed for typing Cucumber mosaic virus (Deyong et al. 2005) and Plum pox virus (Pasquini et al. 2008), enabling the distinction of the 3 existing serotypes or 4 strains respectively. An alternative low technology approach is based in PCR-ELISA (Fig. 3). In this case the PCR amplicons are labelled with Digoxigenin and detected by an anti-Digoxigenin enzyme conjugate. The specific capture probes are biotinylated and immobilized in the wells of an ELISA plate by a streptavidin coating. The hybridization and the detection procedures occur in the same ELISA plate. A great advantage is that the whole procedure uses the equipment usually existing in diagnostic laboratories and can thus be easily introduced in routine. Successful examples of this approach have been developed for typing seven phylogenetic groups of Citrus tristeza virus (Nolasco et al. 2009) or five phylogenetic groups of GLRaV-3 (Gouveia et al. 2009).

\section{Crop-oriented diagnosis: multiplexing and probe arrays}

Convergence into a single methodological platform, i.e nucleic acids based diagnosis, led to the concept of crop-oriented diagnosis, i.e. a unique device or system that could be used for the detection of all the relevant pathogens of a crop. The point of departure for this development is to multiplex the PCR reaction, i.e. include in the same reaction all the primers and probes necessary for detection of several pathogens; each probe is marked with a unique fluorophore which enables the specific pathogen identification. However, the limitations of real time PCR start surfacing here. The technique by itself is not enough robust to support the degree of multiplexing needed in the reaction tube and the fluorescence detection systems are limited to a reduced number of dyes that can be used simultaneously. In practice the higher degree of multiplexing that can be found in the literature is four targets. However, in practice, due to competition between primers this is often reduced to two targets if a good dynamic range is required (Mumford et al. 2006).

Following the development of microarrays for gene expression studies, attempts have been made to implement their use in diagnosis. An initial strategy was to use an array of capture probes specific for the genes of the major pathogens of a crop immobilized in an ordered fashion over a solid phase surface, frequently a silanized microscope glass slide. The array is probed with fluorescently labelled DNA or cDNA obtained from the samples to be tested, resulting in a localized hybridization if the correspondent pathogen was present. The hybridization spots are detected by a fluorescence microscope or a scanner. Due to the enormous number of probing spots that can be deposited in a microarray this seemed a very attracting methodology with an almost unlimited power for parallel analysis. Diverse aspects of this technology were studied in the scope of the COST action 853 "Agricultural Biomarkers for array technology" which extended from 2001 to 2007. However, without any amplification stage, microarrays soon proved to be not very sensitive (Vora et al. 2008) and only a few have been developed, for example for potato viruses (Boonham et al. 2003; Sip et al. 2010) or Genetically Modified Organisms (Prins et al. 2008). An aspect that has been extensively discussed is the need of an amplification step before hybridization to increase 
sensitivity. This amplification step can be easily included if the pathogens targeted have a conserved gene for which generic primers are designed, as for the 16S rRNA gene for prokaryotes or mitochondrial genes for eukaryotes. However this is not feasible for viruses from unrelated taxonomic groups due to the inexistence of conserved genes. This issue was overcome by uncoupling the specific target recognition from amplification. One strategy has been to use Padlock probes - also known as circularizable probes - (Thomas et al. 1999) for target recognition. These probes have two pathogen specific regions at the 5 and 3' ends that come together upon hybridization to the target and are ligated by a DNA ligase. The resulting closed circular molecule becomes permanently locked around the target strand, like a padlock, enabling very stringent washings. The probe has also one or two regions between the pathogen specific ones that will function as universal primers in a subsequent amplification by Rolling Circle Amplification or PCR and a zip-code sequence for hybridization with a subset of the probes in the microarray (Fig. 4). This strategy has been used for fungi and nematodes (Szemes et al. 2005; van Doorn et al. 2007). In another strategy, Engel et al. (2010) used a random primed PCR amplification before hybridization in the development of a microarray designed to detect 44 viruses of the grapevine. To avoid the competing simultaneous amplification of host rRNA, those authors used an enriched ds-RNA preparation instead of total RNA. A comparison of available alternatives for amplification prior to hybridization can be found elsewhere (Vora et al. 2004; Vora et al. 2008). A variety of formats for the arrays and equipment for constructing the array and register the signal exist including electronic solutions in which hybridization is monitored through changes in impedance - for a review see Ramanavicius and Ramanaviciene (2007). However the diversity of existing formats does not help to decrease the price of the equiment and the transfer of microarrays for routine diagnosis is not at close sight.

Alternatively, an affordable and very simple solution that overcomes the multiplexing problems and low sensitivity of microarrays has recently been introduced, called Low-density arrays (LDA). These arrays are just 96- or 384-well PCR plates in which the primers and probes for real-time PCR for each target are deposited and dried in the surface of single wells. PCR reaction mixtures are re-constituted just prior to amplification by adding the common master mix and the template. A great advantage of the method is the easy and immediate transfer of already validated real time PCR protocols to this format, the low technology involved in preparing the arrays and the possibility to continue using the already existent real-time machines. In an assay exemplified for Grapevine viruses (Osman et al. 2008) these authors were able to simultaneously detect 13 viruses.

\section{Non-targeted diagnosis: a new paradigm}

The above detection methods only provide an answer regarding the presence of a certain pathogens for which there is an a priori suspicion and require the previous existence of specific reagents. Those methods cannot provide an answer to the generic question: is this plant infected (with any pathogen)? Some, now classic, techniques as electron microscopy or ds-RNA analysis could answer this question for some viruses but have a limited throughput and lack of sensitivity. New techniques are now surfacing.

For geminiviruses or other pathogens with a closed circular ss-DNA genome a generic method relying in random primed Rolling Circle Amplification followed by enzymatic restriction was developed by Haible et al. (2006). Linear double-stranded monomers can be obtained by restriction digestion or sequenced. This enables the 
identification of known agents or discovery of novel ones with a ss-DNA circular genome.

DNA bar coding is a metagenomic approach which relies in sequencing a gene or a part of the genome which is sufficiently conserved among a large group of taxa to allow the design of general primers, but also sufficiently variable among the species to allow for their identification by nucleotide alignment. From a practical point of view the sample under analysis is amplified by PCR with the general primers and sequenced. The sequences obtained are compared with a dataset and the existing pathogens are identified. Defining the region to be amplified and generating the large dataset is an enormous task that has required a cooperative effort among researchers. The bar code datasets may also be used to design probes for microarrays, thus avoiding the sequencing step. Bar codes are further developed in a companion paper in this series. The high variability that exists in viruses excludes them from this approach.

Next Generation sequencing, Deep sequencing or Massively parallel sequencing (names differ according to the fabricant of the equipment) are automated sequencing platforms which substitute the time consuming in vivo cloning by an automated in vitro system. Thousands of random sequences representing multiple coverage of every DNA molecule are produced in a few hours. By including an initial step of unspecific cDNA synthesis these platforms have been used as a metagenomic approach for RNA viruses. To avoid host RNAs, ds-RNA is used instead of total RNA resulting in an enrichment of viral sequences. This metagenomic approach has been demonstrated for the first time for animal viruses (Cox-Foster et al. 2007) and more recently to plant viruses in a model plant (Adams et al. 2009) or in grapevine (Al Rwahnih et al. 2009; Coetzee et al. 2009; Engel et al. 2009). Interestingly in all the cases new viral agents were found. An example of the information retrieved by such analysis is presented in Fig. 5. Additionally, this approach can be used to furnish the proof that only a certain pathogen is associated with a particular disease, as shown for Candidatus Liberibacter asiaticus, the putative agent of Huanglongbing in citrus (Tyler et al. 2009).

Massive sequencing may also be focused in short RNAs, in the size of 20-30 nt. It is now known that, as part of a defense system, plants respond to virus infection by generating short-interfering RNAs (siRNAs) ranging from $21 \mathrm{nt}$ to $24 \mathrm{nt}$ which are complementary to viral genomes and guide a specific degradation of viral genomes. An important feature of this system is that part of these siRNA are replicated by host RNA polymerases and systemically delivered through parts of the plant not yet infected. Screening for virus related si-RNA appears thus as an alternative for viruses which replicate at low levels or that have an uneven distribution (Kreuze et al. 2009; Pantaleo et al. 2009).

\section{Conclusions}

Except for a few jumps in technology like massive parallel sequencing, the last ten years did not see the richness of introduction of novel technologies in diagnosis like the previous period. Instead, phytodiagnosis developed through the consolidation of existing paradigms - target-oriented, crop-oriented or the new, non-targeted pathogen detection. The molecular, pathogen-targeted or crop-oriented diagnosis does not depend anymore on the existence of a sophisticated and expensive laboratory environment. Unfortunately a sound comparison of the sensitivity and specificity of existing methods is in most cases still lacking. Availability of this data would help deciding which kind of technology should be installed in particular scenarios in developing countries. 


\section{ACKNOWLEDGEMENTS}

Some original experimental results presented in this review were obtained in the scope of grant PTDC AGR-GPL/67844/2006 from Fundação para a Ciência e Tecnologia, Portugal.

\section{Literature cited}

Adams, I.P., R.H. Glover, W.A. Monger, R. Mumford, E. Jackeviciene, M. Navalinskiene, M. Samuitiene and N. Boonham. 2009. Next-generation sequencing and metagenomic analysis: a universal diagnostic tool in plant virology. Mol Plant Pathol 10(4): 537-545.

Al Rwahnih, M., S. Daubert, D. Golino and A. Rowhani. 2009. Deep sequencing analysis of RNAs from a grapevine showing Syrah decline symptoms reveals a multiple virus infection that includes a novel virus. Virology 387(2): 395-401.

Algar, W.R., A.J. Tavares and U.J. Krull. 2010. Beyond labels: a review of the application of quantum dots as integrated components of assays, bioprobes, and biosensors utilizing optical transduction. Anal Chim Acta 673(1): 1-25.

Bonants, P., M. Szemes, R. van Doorn and C.D. Schoen. 2007. Diagnostic application of padlock probes - Multiplexdetection of plant pathogens using universal microarrays. pp 63-70 in Agricultural Biomarkers for Array Technology. J. E. Frey and F. Pasquer, Ed., Agroscope Changins-Wadensil ACW.

Boonham, N., K. Walsh, P. Smith, K. Madagan, I. Graham and I. Barker. 2003. Detection of potato viruses using microarray technology: towards a generic method for plant viral disease diagnosis. J Virol Methods 108(2): 181-187.

Boubourakas, I.N., S. Fukuta and P.E. Kyriakopoulou. 2009. Sensitive and rapid detection of peach latent mosaic viroid by the reverse transcription loop-mediated isothermal amplification. J Virol Methods 160(1-2): 63-68.

Coetzee, B., M.-D. Freeborough, H.J. Maree, J.-M. Celton, D.J.G. Rees and J. Burger. 2009. Virome of a vineyard: Ultra deep sequence analysis of diseased grapevines. pp 216-217 in Extended abstracts 16th Meeting of ICGV, Dijon, France 31 Aug-4 Sept 2009.

Cox-Foster, D.L., S. Conlan, E.C. Holmes, G. Palacios, J.D. Evans, N.A. Moran, P.L. Quan, T. Briese, M. Hornig, D.M. Geiser, V. Martinson, D. vanEngelsdorp, A.L. Kalkstein, A. Drysdale, J. Hui, J. Zhai, L. Cui, S.K. Hutchison, J.F. Simons, M. Egholm, J.S. Pettis and W.I. Lipkin. 2007. A metagenomic survey of microbes in honey bee colony collapse disorder. Science 318(5848): 283-287.

Curtis, K.A., D.L. Rudolph and S.M. Owen. 2009. Sequence-specific detection method for reverse transcription, loop-mediated isothermal amplification of HIV-1. Journal of Medical Virology 81(6): 966-972.

Deyong, Z., P. Willingmann, C. Heinze, G. Adam, M. Pfunder, B. Frey and J.E. Frey. 2005. Differentiation of Cucumber mosaic virus isolates by hybridization to oligonucleotides in a microarray format. J Virol Methods 123(1): 101-108.

Engel, E.A., P.F. Escobar, P.A. Rivera and P.D. Valenzuela. 2009. Grapevine viruses in Chile: Multi-parallel detection based on metagenomic strategies. pp 38-39 in Extended abstracts 16th Meeting of ICGV, Dijon, France 31 Aug-4 Sept 2009. 
Engel, E.A., P.F. Escobar, L.A. Rojas, P.A. Rivera, N. Fiore and P.D. Valenzuela. 2010. A diagnostic oligonucleotide microarray for simultaneous detection of grapevine viruses. J Virol Methods 163(2): 445-451.

Fukuta, S., S. Kato, K. Yoshida, Y. Mizukami, A. Ishida, J. Ueda, M. Kanbe and Y. Ishimoto. 2003. Detection of tomato yellow leaf curl virus by loop-mediated isothermal amplification reaction. J Virol Methods 112(1-2): 35-40.

Fukuta, S., K. Ohishi, K. Yoshida, Y. Mizukami, A. Ishida and M. Kanbe. 2004. Development of immunocapture reverse transcription loop-mediated isothermal amplification for the detection of tomato spotted wilt virus from chrysanthemum. $\mathrm{J}$ Virol Methods 121(1): 49-55.

Goto, M., E. Honda, A. Ogura, A. Nomoto and K.-I. Hanaki. 2009. Colorimetric detection of loop mediated isothermal amplificationreaction by using hydroxy naphthol blue. Biotechniques 46: 167-172.

Gouveia, P., F. Esteves, M. Teixeira Santos, F. Fonseca, J.E. Eiras-Dias and G. Nolasco. 2009. Assessment of GLRaV-3 variants occuring in Portuguese grapevine varieteies according to the coat protein gene. pp 316-317 in Extended abstracts 16th Meeting of $I C G V$, Dijon, France, 31 Aug- 4 Sept 2009.

Haible, D., S. Kober and H. Jeske. 2006. Rolling circle amplification revolutionizes diagnosis and genomics of geminiviruses. J Virol Methods 135(1): 9-16.

Jeong, Y.J., K. Park and D.E. Kim. 2009. Isothermal DNA amplification in vitro: the helicase-dependent amplification system. Cell Mol Life Sci 66(20): 3325-3336.

Kaneko, H., T. Kawana, E. Fukushima and T. Suzutani. 2007. Tolerance of loopmediated isothermal amplification to a culture medium and biological substances. $\mathrm{J}$ Biochem Biophys Methods 70(3): 499-501.

Kennedy, B., K. Arar, V. Reja and R.J. Henry. 2006. Locked nucleic acids for optimizing displacement probes for quantitative real-time PCR. Anal Biochem 348(2): 294-299.

Kikuchi, T., T. Aikawa, Y. Oeda, N. Karim and N. Kanzaki. 2009. A rapid and precise diagnostic method for detecting the Pinewood nematode Bursaphelenchus xylophilus by loop-mediated isothermal amplification. Phytopathology 99(12): 1365-1369.

Kreuze, J.F., A. Perez, M. Untiveros, D. Quispe, S. Fuentes, I. Barker and R. Simon. 2009. Complete viral genome sequence and discovery of novel viruses by deep sequencing of small RNAs: a generic method for diagnosis, discovery and sequencing of viruses. Virology 388(1): 1-7.

Kuan, C.P., M.T. Wu, Y.L. Lu and H.C. Huang. 2010. Rapid detection of squash leaf curl virus by loop-mediated isothermal amplification. J Virol Methods.

Kubota, R., B.G. Vine, A.M. Alvarez and D.M. Jenkins. 2008. Detection of Ralstonia solanacearum by loop-mediated isothermal amplification. Phytopathology 98(9): 1045-1051.

Kutyavin, I.V., I.A. Afonina, A. Mills, V.V. Gorn, E.A. Lukhtanov, E.S. Belousov, M.J. Singer, D.K. Walburger, S.G. Lokhov, A.A. Gall, R. Dempcy, M.W. Reed, R.B. Meyer and J. Hedgpeth. 2000. 3'-minor groove binder-DNA probes increase sequence specificity at PCR extension temperatures. Nucleic Acids Res 28(2): 655661.

Marras, S.A.E. 2006. Selection of fluorophore and quencher pairs for fluorescent nucleic acid hybridization probes. pp 3-16 in Fluorescent energy transfer nucleic acid probes. V. V. Didenko, Ed. Totowa, New Jersey, Humana Press.

Mori, Y., M. Kitao, N. Tomita and T. Notomi. 2004. Real-time turbidimetry of LAMP reaction for quantifying template DNA. J Biochem Biophys Methods 59(2): 145-157. 
Mori, Y., K. Nagamine, N. Tomita and T. Notomi. 2001. Detection of loop-mediated isothermal amplification reaction by turbidity derived from magnesium pyrophosphate formation. Biochem Biophys Res Commun 289(1): 150-154.

Mumford, R., N. Boonham, J. Tomlinson and I. Barker. 2006. Advances in molecular phytodiagnostics - new solutions for old problems. European Journal of Plant Pathology 116: 1-19.

Nagamine, K., T. Hase and T. Notomi. 2002. Accelerated reaction by loop-mediated isothermal amplification using loop primers. Mol Cell Probes 16(3): 223-229.

Nie, X.Z. 2005. Reverse transcription loop-mediated isothermal amplification of DNA for detection of Potato virus Y. plant disease 89(6): 605-610.

Nolasco, G., C. Santos, G. Silva and F. Fonseca. 2009. Development of an asymmetric PCR-ELISA typing method for citrus tristeza virus based on the coat protein gene. $\mathrm{J}$ Virol Methods 155(2): 97-108.

Notomi, T., H. Okayama, H. Masubuchi, T. Yonekawa, K. Watanabe, N. Amino and T. Hase. 2000. Loop-mediated isothermal amplification of DNA. Nucleic Acids Res 28(12): E63.

Ortiz, E., G. Estrada and P.M. Lizardi. 1998. PNA molecular beacons for rapid detection of PCR amplicons. Mol Cell Probes 12(4): 219-226.

Osman, F., C. Leutenegger, D. Golino and A. Rowhani. 2008. Comparison of low-density arrays, RT-PCR and real-time $\operatorname{TaqMan}((\mathrm{R}))$ RT-PCR in detection of grapevine viruses. Journal of Virological Methods 149(2): 292-299.

Pantaleo, V., P. Saldarelli, L. Miozzi, A. Giampetruzzi, A. Gisel, S. Moxon and T. Dalmay, Burgyan, J. 2009. Deep sequencing analysis of viral short RNAs from Pinot Noir clone ENTAV 115. pp 218-219 in Extended abstracts of 16th Meeting of ICGV, Dijon, France 31 Aug-4 Sept 2009.

Pasquini, G., M. Barba, A. Hadidi, F. Faggioli, R. Negri, I. Sobol, A. Tiberini, K. Caglayan, H. Mazyad, G. Anfoka, M. Ghanim, M. Zeidan and H. Czosnek. 2008. Oligonucleotide microarray-based detection and genotyping of Plum pox virus. J Virol Methods 147(1): 118-126.

Prins, T.W., J.P. van Dijk, H.G. Beenen, A.A. Van Hoef, M.M. Voorhuijzen, C.D. Schoen, H.J. Aarts and E.J. Kok. 2008. Optimised padlock probe ligation and microarray detection of multiple (non-authorised) GMOs in a single reaction. BMC Genomics 9: 584.

Ramanavicius, A. and A. Ramanaviciene. 2007. Electrochemical methods for DNA and protein detection. pp 25-34 in Agricultural Biomarkers for Array Technology. J. E. Frey and F. Pasquer, Ed., Agroscope Changins-Wadensil ACW.

Rigano, L.A., M.R. Marano, A.P. Castagnaro, A.M. Do Amaral and A.A. Vojnov. 2010. Rapid and sensitive detection of Citrus Bacterial Canker by loop-mediated isothermal amplification combined with simple visual evaluation methods. BMC Microbiol 10: 176.

Sip, M., D. Bystricka, S. Kmoch, L. Noskova, H. Hartmannova and P. Dedic. 2010. Detection of viral infections by an oligonucleotide microarray. J Virol Methods 165(1): 64-70.

Szemes, M., P. Bonants, M. de Weerdt, J. Baner, U. Landegren and C.D. Schoen. 2005. Diagnostic application of padlock probes--multiplex detection of plant pathogens using universal microarrays. Nucleic Acids Res 33(8): e70. 
Thomas, D.C., G.A. Nardone and S.K. Randall. 1999. Amplification of padlock probes for DNA diagnostics by cascade rolling circle amplification or the polymerase chain reaction. Arch Pathol Lab Med 123(12): 1170-1176.

Tomlinson, J.A., I. Barker and N. Boonham. 2007. Faster, simpler, more-specific methods for improved molecular detection of Phytophthora ramorum in the field. Applied and Environmental Microbiology 73(12): 4040-4047.

Tyler, H.L., L.F. Roesch, S. Gowda, W.O. Dawson and E.W. Triplett. 2009. Confirmation of the sequence of 'Candidatus Liberibacter asiaticus' and assessment of microbial diversity in Huanglongbing-infected citrus phloem using a metagenomic approach. Mol Plant Microbe Interact 22(12): 1624-1634.

van Doorn, R., M. Szemes, P. Bonants, G.A. Kowalchuk, J.F. Salles, E. Ortenberg and C.D. Schoen. 2007. Quantitative multiplex detection of plant pathogens using a novel ligation probe-based system coupled with universal, high-throughput real-time PCR on OpenArrays. BMC Genomics 8: 276.

Varga, A. and D. James. 2006. Use of reverse transcription loop-mediated isothermal amplification for the detection of Plum pox virus. Journal of Virological Methods 138(1-2): 184-190.

Vincent, M., Y. Xu and H. Kong. 2004. Helicase-dependent isothermal DNA amplification. EMBO Rep 5(8): 795-800.

Vora, G.J., C.E. Meador, G.P. Anderson and C.R. Taitt. 2008. Comparison of detection and signal amplification methods for DNA microarrays. Mol Cell Probes 22(5-6): 294-300.

Vora, G.J., C.E. Meador, D.A. Stenger and J.D. Andreadis. 2004. Nucleic acid amplification strategies for DNA microarray-based pathogen detection. Appl Environ Microbiol 70(5): 3047-3054.

Zhao, K., Y. Liu and X. Wang. 2010. Reverse transcription loop-mediated isothermal amplification of DNA for detection of Barley yellow dwarf viruses in China. J Virol Methods. 


\section{$\underline{\text { Tables }}$}

Table 1. Features of some amplification techniques

\begin{tabular}{|c|c|c|c|c|c|c|c|c|c|c|}
\hline & PCR & $\mathrm{LCR}^{11}$ & G-LCR & NASBA & 3SR & SDA & TMA & LAT & $\mathrm{RCA}$ & LAMP \\
\hline \multicolumn{11}{|l|}{ Enzymes } \\
\hline DNA polymerase & + & - & + & - & - & + & - & - & + & + \\
\hline RNA polymerase & - & - & - & + & + & - & + & + & - & - \\
\hline DNA ligase & - & + & + & - & - & - & - & + & - & - \\
\hline RTase $^{2}$ & $+1-$ & $+/-$ & $+1-$ & + & + & $+1-$ & + & + & - & $+1-$ \\
\hline RNAse H & - & - & - & + & + & - & - & + & - & - \\
\hline Restriction Enz. & - & - & - & - & - & + & - & - & - & - \\
\hline Primers or probes & 2 & 4 & 4 & 2 & 2 & 4 & 2 & 2 & 1 or 2 & 4 or 6 \\
\hline Isothermal & - & - & - & + & + & + & + & + & + & + \\
\hline
\end{tabular}

(1) - LCR: Ligase Chain reaction; G-LCR: Gap Ligase Chain reaction; SDA: Strand Displacement Amplification; LAT: Ligation-Activated Transcription; 3SR: Self Sustained Sequence Replication; RCA: rolling Circle Amplification; LAMP: Loop-mediated Isothermal Amplification; TMA: Transcriptionmediated Amplification

(2) -Reverse Transcriptase is needed only for RNA templates (+ / -) or mandatory, independently of the nature of the template $(+)$

Table 2. Some fluorescent nucleic acid hybridization probes and their mechanism of energy transfer

\begin{tabular}{lcc}
\hline Fluorescent hybridization probes & \multicolumn{2}{c}{ Energy transfer } \\
& FRET $^{1}$ & Contact quenching $^{2}$ \\
\hline Adjacent probes & + & - \\
Amplifluor primers & $?$ & + \\
Cyclicons & $?$ & + \\
Duplex scorpion primers & - & + \\
HyBeacons & - & + \\
Molecular beacon probes & $?$ & + \\
5'-nuclease probes (TaqMan ${ }^{\circledR}$ probes) & + & $?$ \\
Scorpion primers & $\boldsymbol{?}$ & + \\
Strand-displacement probes (Yin-Yang probes) & $?$ & + \\
Wavelength-shifting molecular beacon probes & $?$ & + \\
\hline
\end{tabular}

Source: Marras (2006)

(1) - Quenching of the fluorophore by FRET can occur if the fluorophore and quencher pair have spectral overlap and remain within sufficient distance of each other for efficient energy transfer to occur.

(2) - Quenching of the fluorophore by contact quenching can occur if the fluorophore comes in close proximity to the quencher molecule or to a nucleotide, owing to internal hybrid formation within the probe 


\section{Figures}
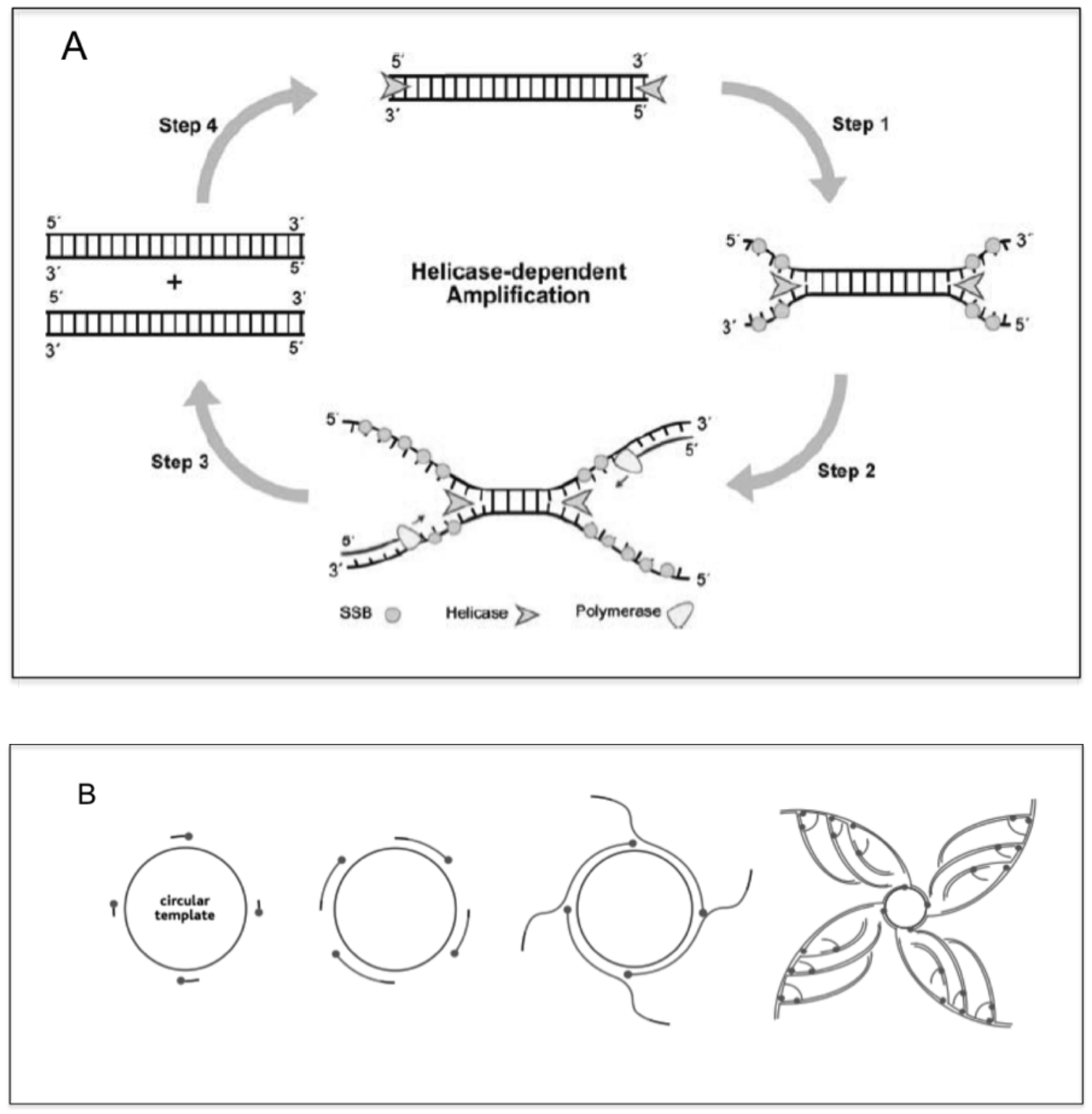

Fig. 1. Recent amplification systems. Panel A. Helicase Dependent Amplification (HDA); Helicase unwinds the template extremities, single-stranded binding (SSB) proteins maintain the strands opened and allow primers to anneal; the DNA polymerase extends the primers and new two molecules are formed; being isothermical the process is continuous. Source: Jeong et al. (2009). Panel B. Rolling circle mechanism with random primers. Primers anneal in several places of the single stranded template and are extended by action of DNA polymerase; the strand displacement activity of the DNA polymerase continuously frees new portions of single strand DNA to which new primers anneal originating a mass of full-length ds-DNA (adapted from GE Healthcare Templiphi brochure). If instead of random primers only one specific forward primer is included in the reaction the result is a multimeric linear ss-DNA which is amplified aritmethically. If the reverse primer is also included the amplification is exponential and originates dsDNA multimers. 

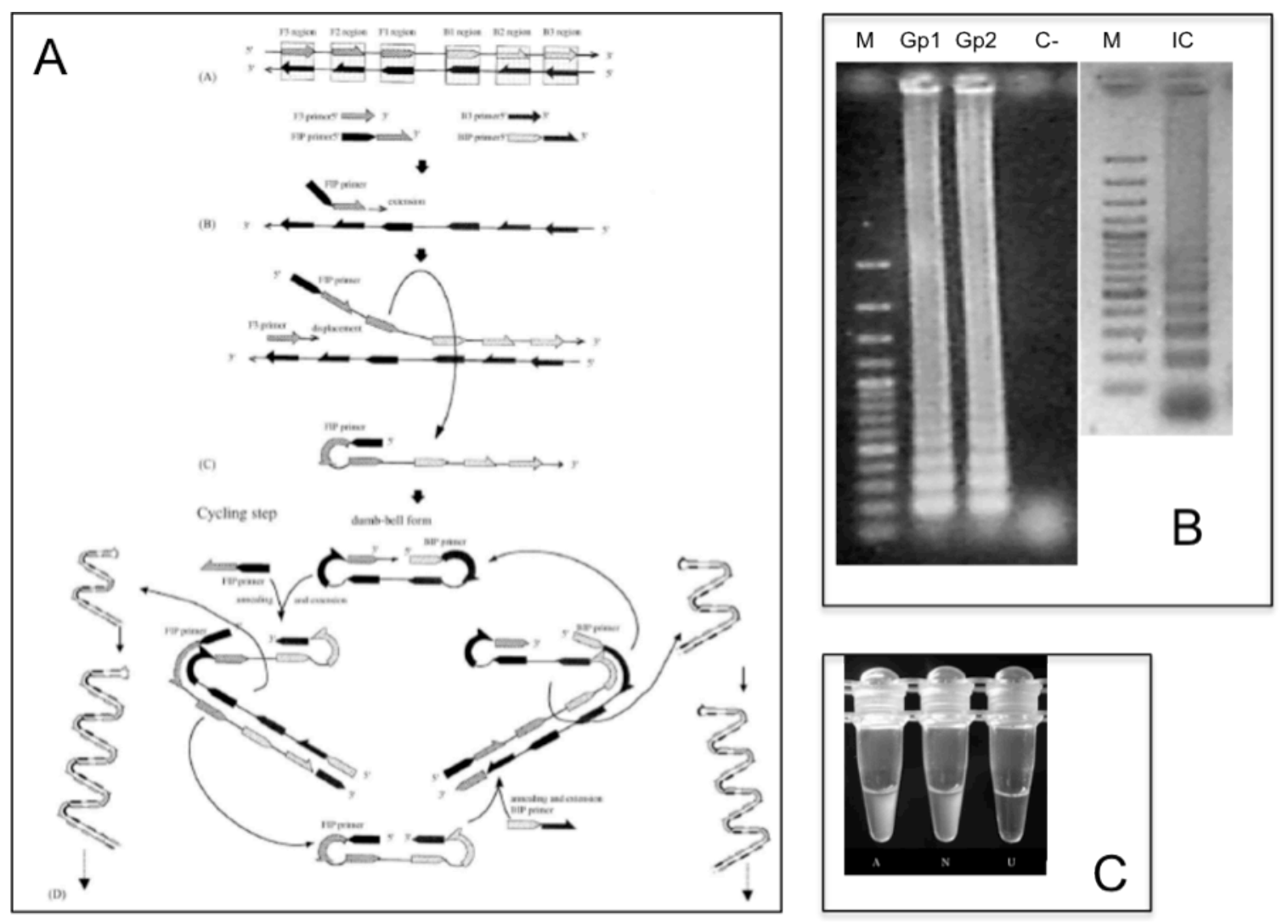

Fig. 2. Some aspects of LAMP. Panel A, amplification scheme. (A) primers; (B) first extension by FIP primer followed by strand displacement by extension of F3 primer; (C) BIP primer anneals to the displaced strand and is extended (not shown), resulting in the dumbbell shape which is central for the cyclic amplification process; (D) cyclic amplification originating a collection of multimeric sequences with diverse sizes (adapted from Fukuta et al. (2004). Panel B, electrophoresis of LAMP products obtained from GLRaV-3 showing the collection of different sized molecules; Gp1 and Gp2 amplification of isolates from phylogenetic groups 1 and 2, IC - amplification following immunocapture, $\mathrm{M}-100$ bp ladder, $\mathrm{C}$ - negative control. Panel C, whitish precipitate of magnesium pyrophosphate formed in tubes where a successful amplification of TYLCV took place (leftmost tubes); the samples analysed were tomato tissue ground in $\mathrm{NaOH}$; source: Fukuta et al. (2003) 

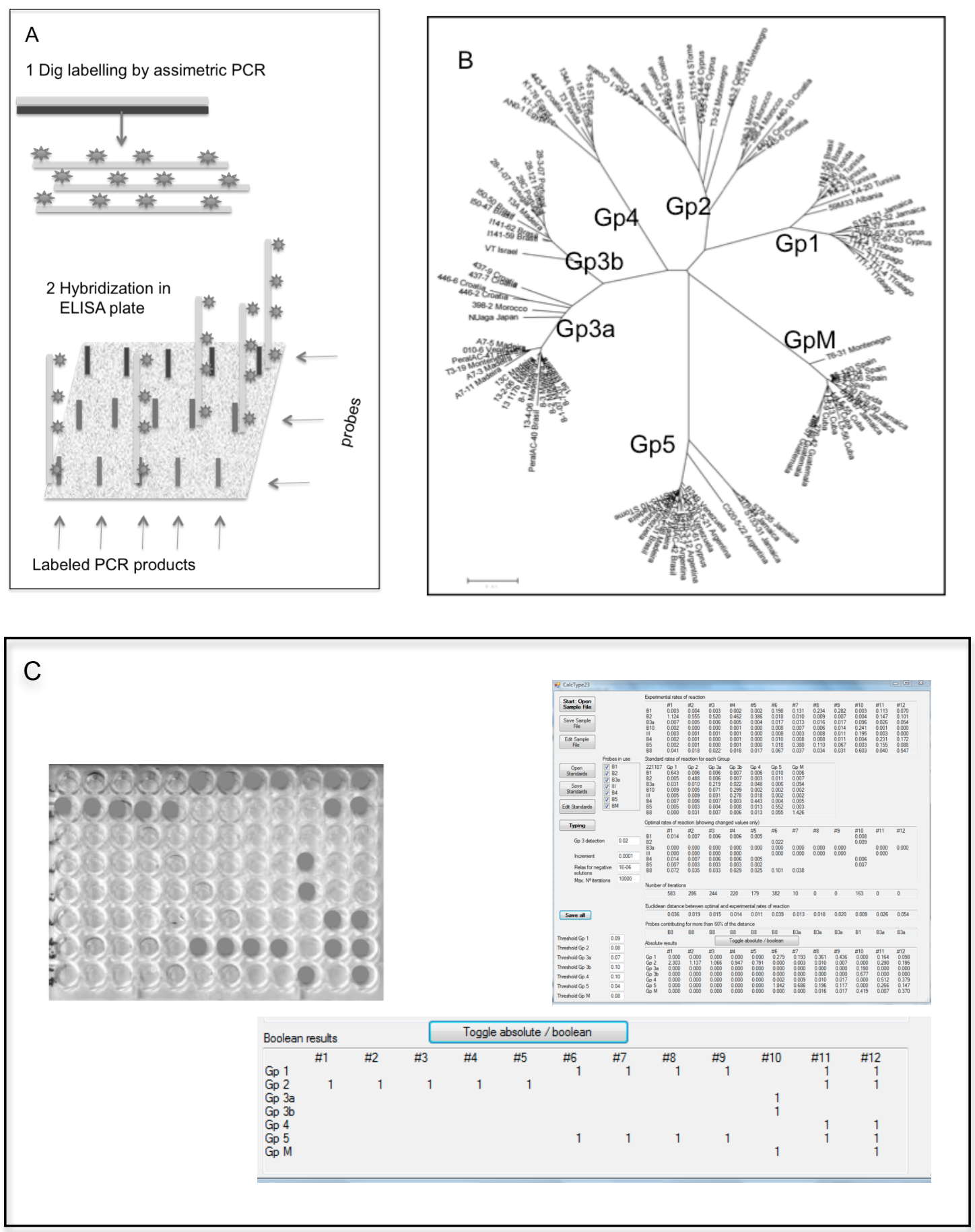

Fig. 3. Asymmetric PCR-ELISA Typing. Panel A, scheme of labelling of PCR products with Digoxigenin by an asymmetric PCRreaction and hybridization in a array of probes made in an ELISA plate. Panel B, Phylogenetic tree of the coat protein gene of Citrus tristeza virus used to define the 7 groups and probes. Panel C, Example of the final results: after adding the ELISA substrate the reaction rate of the hydrolysis is computed and by software comparison with a panel of values of standard rates for each group the strain composition of the sample is inferred; notice that some probes react with more than one group but at different rates and in the ELISA plate shown only the more intense reactions are conspicuous. Adapted from Nolasco et al. (2009) 

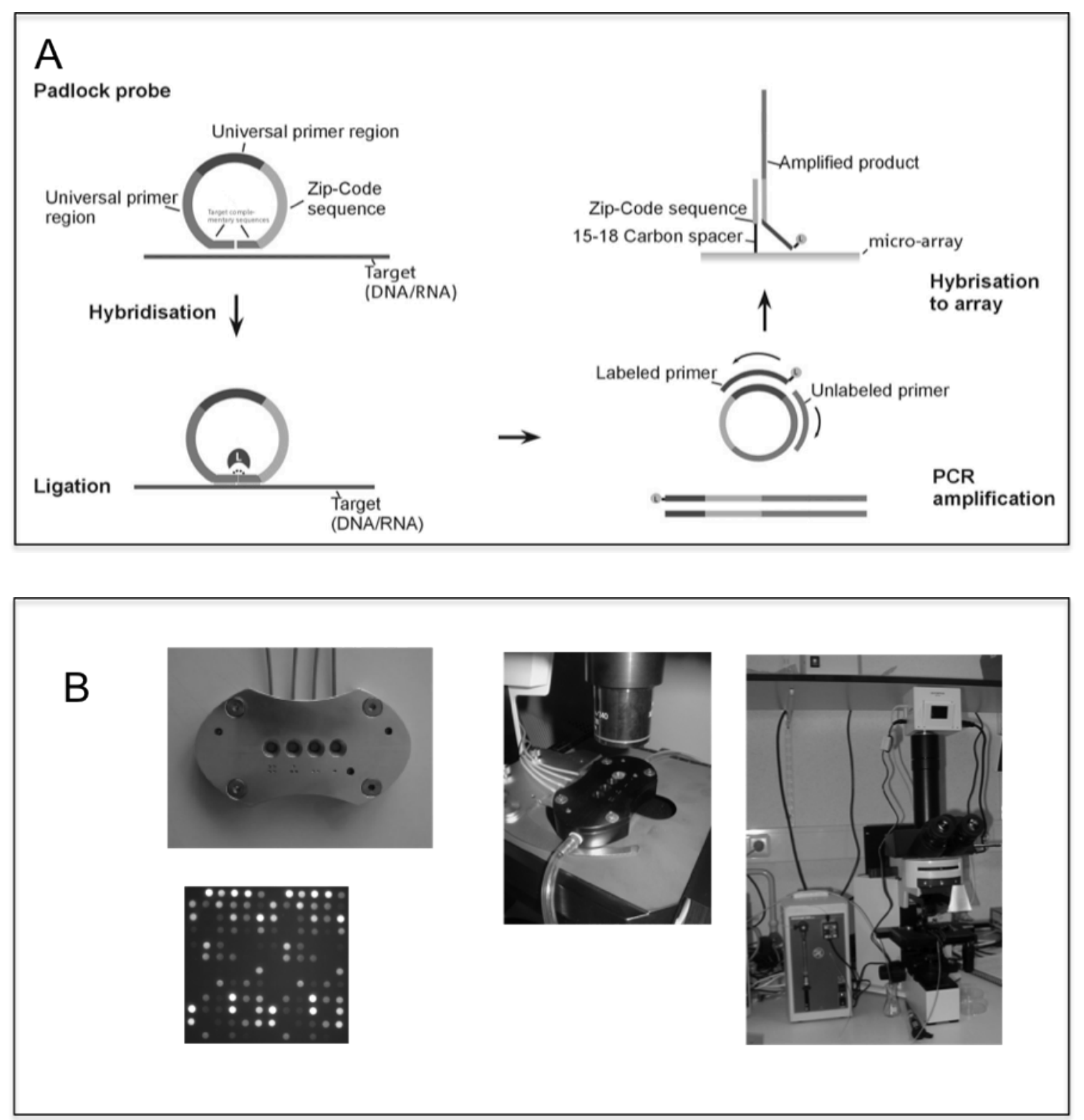

Fig. 4. Microarrays. Panel A, schematic view for padlock probe hybridisation, ligation and PCR amplification of circularised probes followed by hybridisation to a microarray system; the padlock probe consists of two target complementary sequences, two universal forward and reverse primers sequences, and a unique identifier sequence (Zip-code). Panel B, view of components of a laboratory made system, hybridization chamber, fluorescence microscope and fluorescent dots obtained in microscope slide array. Source: Bonants et al. (2007). 
Fragment counts for viral species identified in the BLASTN analysis of the total data set.

\begin{tabular}{|c|c|c|}
\hline \multirow[t]{2}{*}{ Organism name } & \multicolumn{2}{|c|}{ Total hits } \\
\hline & $\mathrm{I}$ & II \\
\hline \multicolumn{3}{|l|}{$\bar{A}$} \\
\hline Rupestris stem pitting-assaciated virus & 46,029 & 46,029 \\
\hline Grapevine rupestris vein-feathering virus & 9791 & 9697 \\
\hline Grapevine Syrah virus-1 & - & 1527 \\
\hline Grapevine leafroll-associated virus-9 & 16 & 16 \\
\hline Hop stunt viroid & 13 & 13 \\
\hline Grapevine yellow speckle viroid & 5 & 5 \\
\hline Australian grapevine viroid & 4 & 4 \\
\hline \multicolumn{3}{|l|}{ B } \\
\hline Grapevine asteroid mosaic-assaciated virus & 113 & 27 \\
\hline Grapevine fleck vinus & 11 & 0 \\
\hline \multicolumn{3}{|l|}{$c$} \\
\hline Maize rayado fino virus & 55 & 12 \\
\hline Citrus sudden death-associated virus & 40 & 21 \\
\hline Oat blue dwarf virus & 34 & 2 \\
\hline Okra mosaic virus & 14 & 4 \\
\hline Kennedya yellow mosaic virus & 2 & 1 \\
\hline Nemesia ring necrosis virus & 2 & 2 \\
\hline Erysimum latent virus & 1 & 1 \\
\hline Tumip yellow mosaic virus & 1 & 1 \\
\hline \multicolumn{3}{|l|}{ D } \\
\hline Cucumber mosaic virus & 1 & 1 \\
\hline Sacbrood vinus & 1 & 1 \\
\hline Total & 56,133 & 57,365 \\
\hline
\end{tabular}

Column I, initial analysis; column II, after the addition of the GSyV-1 sequence to the query database. $(A)$ Viruses and viroids expected in grapevine, the presence of which was confirmed by specific PCR analysis. (B) Viruses expected to be found in grapevine, but not confirmed as present in the sample by specific PCR analysis. (C, D) Viruses not expected to be found in grapevine (not tested by PCR analysis); (C) Tymoviridae, (D) others.

Fig. 5. Non-targeted diagnosis. Example of the information retrieved from a single symptomatic (red leaves, swelling and wood necrosis at the graft union, stem pitting above the graft union) grapevine plant by massive parallel sequencing. Notice that besides a new virus (Grapevine syrah virus 1) several other viruses were not expected to be found in grapevine. Source: Al Rwahnih et al. (2009) 